$\mathrm{BMB}$

\title{
Drosophila as a model for unfolded protein response research
}

\author{
Hyung Don Ryoo * \\ Department of Cell Biology, New York University School of Medicine, New York, NY 10016, USA
}

\begin{abstract}
Endoplasmic Reticulum (ER) is an organelle where most secretory and membrane proteins are synthesized, folded, and undergo further maturation. As numerous conditions can perturb such ER function, eukaryotic cells are equipped with responsive signaling pathways, widely referred to as the Unfolded Protein Response (UPR). Chronic conditions of ER stress that cannot be fully resolved by UPR, or conditions that impair UPR signaling itself, are associated with many metabolic and degenerative diseases. In recent years, Drosophila has been actively employed to study such connections between UPR and disease. Notably, the UPR pathways are largely conserved between Drosophila and humans, and the mediating genes are essential for development in both organisms, indicating their requirement to resolve inherent stress. By now, many Drosophila mutations are known to impose stress in the ER, and a number of these appear similar to those that underlie human diseases. In addition, studies have employed the strategy of overexpressing human mutations in Drosophila tissues to perform genetic modifier screens. The fact that the basic UPR pathways are conserved, together with the availability of many human disease models in this organism, makes Drosophila a powerful tool for studying human disease mechanisms. [BMB Reports 2015; 48(8): 445-453]
\end{abstract}

\section{INTRODUCTION}

It is estimated that approximately a third of all genes in eukaryotes encode secretory or membrane proteins that are synthesized on the rough endoplasmic reticulum $(E R)(1,2)$. Peptides that are synthesized into this organelle first undergo chaperone-assisted folding, and a subset is further modified through glycosylation or oxidation to form disulfide bonds (3). Proteins that fail to undergo proper folding and maturation can be toxic to cells, and underlie many metabolic and degenerative diseases that include diabetes and various forms of neuro-

*Corresponding author. Tel: +1-212-263-7257; Fax: +1-212-2638139; E-mail: hyungdon.ryoo@nyumc.org

http://dx.doi.org/10.5483/BMBRep.2015.48.8.099

Received 21 May 2015

Keywords: Drosophila, Endoplasmic reticulum, IRE1, PERK, Unfolded protein response degeneration $(4,5)$. Eukaryotic cells have evolved a robust Unfolded Protein Response (UPR), which specifically refers to signaling pathways that regulate gene expression in response to ER stress $(6,7)$. Naturally, the regulation of the UPR has been a topic that has drawn significant interest in the field.

Cells can suffer from ER stress for a variety of reasons. Perhaps most obvious are mutations that impair the inherent folding properties of an encoded protein (6). These proteins can cause aggregates, and also have the effect of overwhelming the protein folding machinery. ER is also an organelle that stores high concentrations of $\mathrm{Ca}^{2+}$, which in turn is essential for proper ER function. In fact, the $\mathrm{Ca}^{2+}$ pump inhibitor thapsigargin is frequently used among researchers to impose stress in the ER (8). Calnexin and Calreticulin are examples of $\mathrm{Ca}^{2+}$ binding proteins that are specifically involved in the folding of glycosylated proteins in the ER, and inhibition of glycosylation with tunicamycin similarly interferes with protein folding in this organelle (9). In addition, ER has an oxidizing environment that promotes the formation of disulfide bonds between cysteine residues (10). Many proteins in the ER make stable domain structures only when certain disulfide bonds are formed, and inhibition of cysteine oxidation with reducing agents such as DTT also imposes severe stress in the ER. The rapid elucidation of the UPR pathways was possible, in part, due to the facile ER stress assays based on tunicamycin, thapsigargin and DTT treatment on cultured cells.

The term, UPR, was first coined to describe the transcriptional response to mutant viral protein expression in cultured mammalian cells (6). Such transcriptional response is also observed in Drosophila, as documented in detail in more than a hundred inbred Drosophila species that were fed tunicamycin (11). In recent years, there have been significant efforts to go beyond drug treatment experiments, and determine the physiological role of the UPR in animal development, tissue homeostasis, disease models and lifespan regulation. Drosophila has emerged as a popular model organism for those studies, and here I will discuss the recent advances in this area.

\section{IRE1/XBP1 PATHWAY OF THE UPR}

The UPR pathway was initially dissected in the baker's yeast, Saccharomyces cerevisiae (12). It was first found that IRE1 is an essential mediator of ER chaperone induction after ER stress, as such conditions prompt the activation of this transmembrane signaling protein by forcing oligomer formation

ISSN: 1976-670X (electronic edition)

Copyright (c) 2015 by the The Korean Society for Biochemistry and Molecular Biology

(ㄷ) This is an open-access article distributed under the terms of the Creative Commons Attribution Non-Commercial License (http://creativecommons.org/licenses/by-nc/4.0) which permits unrestricted non-commercial use, distribution, and reproduction in any medium, provided the original work is properly cited. 
$(13,14)$. X-ray crystallography studies revealed that IRE1 has an RNase domain (15). Once activated, IRE1 cleaves the HAC1 mRNA to initiate mRNA splicing. This splicing reaction is completed after the two cleaved mRNA pieces are put together by a tRNA ligase (16). Once the spliced HAC1 mRNA is translated, the encoded protein serves as a transcription factor that induces the expression of ER quality control genes (17).

Mammals have two IRE1 homologs, IRE1alpha and beta, which respond to ER stress by cutting XBP1 mRNA in two specific positions, similar to how yeast IRE1 cuts HAC1 mRNA $(18,19)$ (Fig. 1A). The splicing reaction is completed by the ligation of the two cleaved pieces, and recently, the responsible RNA ligase was identified in mammals and C. elegans (20-22). Drosophila has a single IRE1 gene that cleaves a conserved XBP1 mRNA $(23,24)$, and an uncharacterized homolog of the newly identified mammalian ligase, annotated as CG9987. Both in mammals and Drosophila, BAX Inhibitor-1 overexpression reportedly suppresses IRE1 activity (25). Once the spliced isoform (also known as the RB isoform) of XBP1 is generated, this active transcription factor induces many ER quality genes that help to restore homeostasis (23).

In $S$. cerevisiae, a systematic approach has failed to identify any IRE1 substrates other than the HAC1 mRNA (26). On the other hand, a study based on Drosophila S2 cells revealed that IRE1 cleaves other mRNAs (23), so as to degrade them upon ER stress. More recent work has found that, in general, mRNAs that are normally translated on the rough $\mathrm{ER}$, and therefore accessible to IRE1, are substrates for degradation (27) (Fig. 1A). The initial observation made in S2 cells have now been vali- dated in vivo: Whereas XBP1 mutant mosaic clones did not show obvious developmental defects in the Drosophila eye, IRE1 mutants had defects in the secretion of extracellular materials, which gave rise to a shorter inter-ommatidial distance. One of the targets that contributed to this IRE1-specific phenotype was the mRNA of FATP, which is a target of IRE1-mediated degradation (28). The idea that IRE1 degrades mRNAs associated with the ER has now been validated in other organisms as well, including mammals $(29,30)$. Interestingly, the fission yeast $S$. pombe does not encode an ortholog of HAC1, and a recent study has found that IRE1 helps that organism withstand ER stress primarily by degrading mRNAs associated with the ER (31).

\section{PHYSIOLOGICAL ROLES OF THE IRE1/XBP1 PATHWAY IN Drosophila}

Our understanding of the IRE1/XBP1 pathway is largely based on experiments with cells that were exposed to ER stress causing chemicals, or conditions that express high levels of mutant proteins. Less well understood is the role of IRE1 and XBP1 in vivo, particularly in tissues that are not exposed to exogenous sources of stress. It is clear that IRE1 and XBP1 play important roles even in normally developing tissues, as these are developmentally essential genes. In Drosophila, IRE1 mutants survive only up to the $1^{\text {st }}$ instar stage (32), and XBP1 mutants up to the $2^{\text {nd }}$ instar stage (24).

The precise role of these genes in the developing tissues is only beginning to be understood. In the larval fat body, it was
A

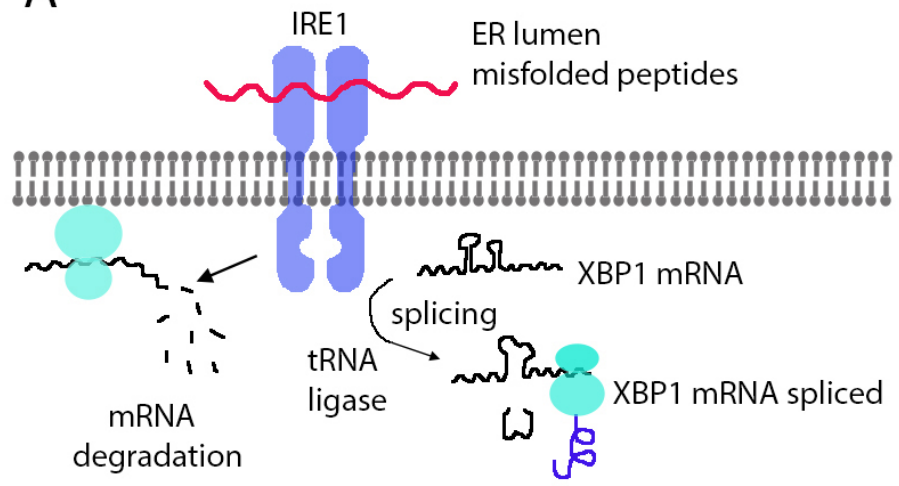

B

XBP1-GFP reporter

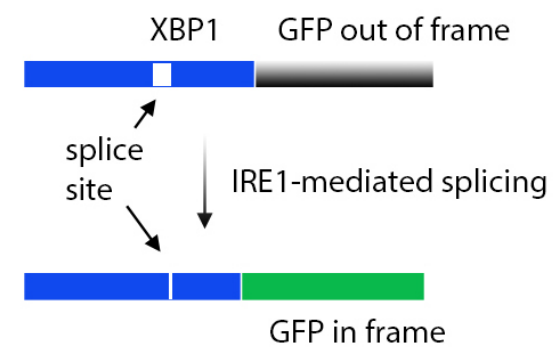

\section{XBP1s $\longrightarrow$ ER quality control genes}

Fig. 1. Regulation and detection of the IRE1/XBP1 pathway. (A) A schematic diagram of the IRE1/XBP1 pathway in Drosophila. IRE1 is an ER stress sensor that directly binds to misfolded peptides in the ER lumen. Upon detecting ER stress, IRE1 activates its RNase domain on the cytoplasmic side. IRE1 works together with a tRNA ligase to catalyze the splicing of XBP1 mRNA. The product of this spliced isoform acts as a transcription factor that induces ER quality control genes. In addition, IRE1 promotes the decay of many mRNAs associated with the ER. (B) The XBP1-GFP reporter used to detect IRE1 activity in vivo. As XBP1 mRNA splicing by IRE1 shifts the reading frame, an XBP1-GFP fusion transgene was designed to have GFP expressed in frame only when IRE1-mediated splicing occurs. 
found that XBP1 mutant cells activate autophagy, indicating that XBP1 is normally active in that tissue to maintain cellular homeostasis (33). The expression pattern and activity of IRE1 and XBP1 suggest possible roles in a number of other tissues. One of those is the gastro-intestinal system. In situ hybridization of xbp 1 most prominently shows high transcript levels in the salivary gland and the intestines, and this has been further confirmed with a reporter under the control of XBP1's upstream sequence $(24,32)$. Aside from the expression pattern, IRE1 activity can be visualized with another reporter, XBP1-GFP $(24,34)$. This reporter takes advantage of the fact that the splicing of XBP1 results in a reading frame shift of that transcript. By placing GFP after the XBP1 sequence in a specific reading frame, GFP is expressed in frame specifically when IRE1-mediated mRNA splicing occurs $(24,34)$ (Fig. 1B). In addition to detecting IRE1 activation in pathological conditions, this reporter can detect ER stress in various tissues, including the larval intestine, fat body, glia, certain neurons and developing photoreceptors $(28,34)$.

A number of adult tissues also show signs of IRE1 activity, including the male reproductive system and the aging adult intestinal stem cells $(34,35)$. The accessory gland normally secretes many seminal fluid proteins, and correlating with this, XBP1-based reporters are active in this tissue $(32,34)$. Further aggravation of such inherent ER stress, either by misexpressing mutant proteins, or by knocking down the ER chaperone BiP, leads to excessive activation of XBP1 splicing and infertility (36). In the adult intestine, XBP1 is required for the proper homeostasis of the epithelial cells, and in its absence the cells build up high levels of reactive oxygen species (ROS), which in turn, signal to promote stem cell hyperproliferation and epithelial dysplasia in the aging fly intestine. Conversely, hyper-activation of the IRE1/XBP1 branch by overexpressing the spliced isoform of XBP1 (XBP1-RB, also known as XBP1s) suppresses ER-stress related phenotypes in the intestinal stem cells $(35,37)$.

\section{DEVELOPMENTAL DEFECTS ASSOCIATED WITH IRE1/XBP1}

Major signaling pathways involve membrane receptors and ligands that are synthesized in the ER, and therefore, dysfunction of the ER may have a broad effect on those pathways. Intriguingly, the Notch signaling pathway in Drosophila appears to be particularly sensitive to the protein-folding environment in the ER. The connection with UPR was first noticed when a genetic screen for a Notch-like phenotype in the fly identified mutations in ero1 $L$, whose normal function is to stimulate disulfide bond formation in the ER. Based on yeast genetic studies, it was assumed at the time that all disulfide bond formation in the ER should be impaired in ero $1 \mathrm{~L} \mathrm{mu-}$ tants, and therefore, loss of this gene would result in a pleiotropic phenotype. However, ero $1 \mathrm{~L}$ mutant cells specifically showed a Notch-like phenotype in Drosophila, with Notch protein accumulation in the $E R$, and activation of the XBP1-GFP reporter (38). Since that study in Drosophila, it has been also determined in mammals that disulfide bonds can form without ero1L, indicating that this gene has assumed more specific roles in metazoans (39).

Other mutations that impair Notch maturation in the ER include mutations in the Catsup gene, a Drosophila homolog of ZIP7 zinc transporter (40), Rumi that encodes an O-glucosyltransferase (41) and pecanex (42). Overexpression of the spliced isoform of XBP1 suppressed the pecanex phenotype (42).

\section{THE PERK/ATF4 PATHWAY IN Drosophila}

In parallel to the IRE1/XBP1 branch, the ER transmembrane kinase PERK is activated in response to ER stress and phosphorylates the translational initiation factor elF2alpha (43) (Fig. 2). The normal role of elF2alpha is to help charge $40 \mathrm{~S}$ ribosomal subunits with initiator methionyl tRNAs, which is essential for translational initiation. Therefore, the inhibitory phosphorylation by PERK attenuates the overall rate of translational initiation. It is generally understood that such reduction in translation helps to alleviate the protein-folding burden of cells, but excessive activation of PERK by gene overexpression in Drosophila tissues can also cause toxicity (44). In addition, such conditions activate downstream signaling pathways. One of the best characterized is that of ATF4, whose synthesis paradoxically increases when elF2alpha is phosphorylated. The underlying mechanism of this intriguing phenomenon has been described in detail elsewhere $(43,45)$. In brief, the unique induction of ATF4 synthesis is possible due to a number of small upstream Open Reading Frames (uORFs) in the 5' UTR (Fig. 2B). The last uORF overlaps with ATF4 in a different reading frame, and therefore, inhibits ATF4 translation in unstressed cells. elF2alpha phosphorylation makes the recognition of this uORF by the $40 \mathrm{~S}$ ribosome inefficient, thereby allowing the opportunity for the main ATF4 ORF to be translated. Once synthesized, ATF4 induces many targets that are involved in stress response. The Drosophila genome encodes single orthologs of PERK and ATF4 $(44,46)$. The latter transcript has uORFs in its $5^{\prime}$ UTR, similar to the homologs in other species. While the ATF4 transcript is widely distributed, the translation only occurs in response to stress $(46,47)$. A Drosophila ATF4 reporter was recently made by fusing the ATF4 $5^{\prime}$ UTR with the coding sequence of dsRed. This reporter is activated in response to mutant membrane protein expression, and also detects stress in a number of normally developing tissues, including the pupal stage photoreceptors (48).

If ATF4 induces many transcripts, only to have their translation blocked by phosphorylated elF2alpha, a robust gene expression response cannot be mounted. In reality, elF2alpha phosphorylation occurs only for a few hours, before being de-phosphorylated to allow mRNA translational initiation. In mammals, such negative feedback occurs through ATF4, and 
A

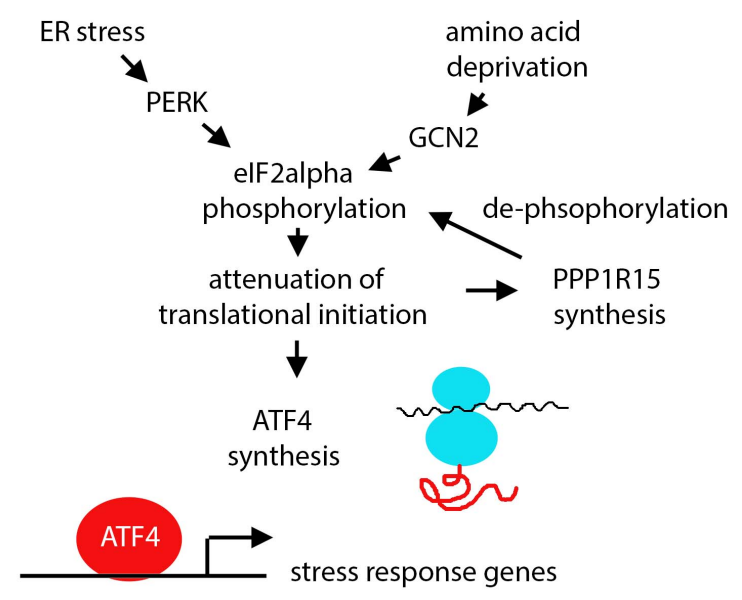

B

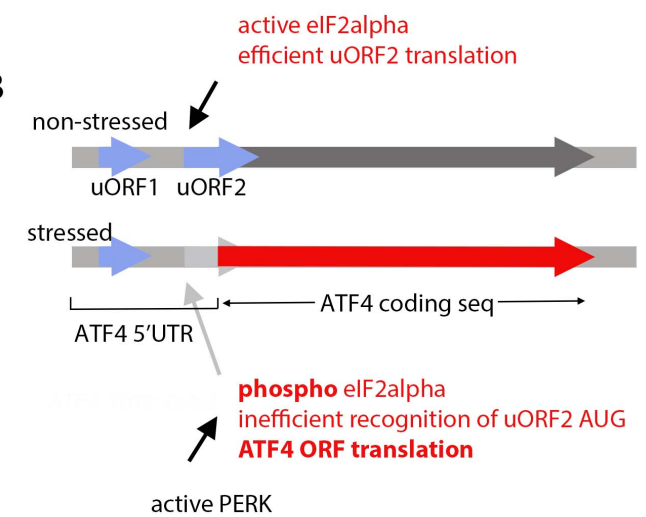

Fig. 2. Regulation of the PERK/ATF4 pathway in Drosophila. (A) A schematic diagram of the pathway initiated by the elF2alpha kinases, PERK and GCN2. These kinases are activated by distinct types of stress and phosphorylate elF2alpha. This results in the overall translation attenuation, but at least two transcripts in Drosophila enhance their translation during such conditions: ATF4 is a transcription factor that induces stress response genes, and PPP1R15 is a phosphatase subunit that helps to de-phosphorylate elF2alpha as a feedback mechanism. (B) uORFs in the 5' UTR allow enhanced ATF4 synthesis under conditions of elF2alpha phosphorylation. ATF4 5' UTR has multiple uORFs, and only two are shown for simplicity. elF2alpha helps to charge 40S ribosomes with initiator methionyl tRNA after the synthesis of uORF1. When elF2alpha is active, $40 \mathrm{~S}$ ribosomes efficiently recognize uORF2 for translation. uORF2 overlaps with ATF4 ORF, and interferes with ATF4 ORF translation. When elF2alpha is phosphorylated, 40S ribosome's ability to recognize the uORF2 is compromised, bypassing its AUG to allow the recognition of the ATF4 ORF.

its downstream transcription factor $\mathrm{CHOP}$, which transcriptionally induces a phosphatase subunit PPP1R15 (also referred to as GADD34) to dephosphorylate elF2lapha. A recent study shows that Drosophila PPP1R15 is also induced by ER stress, but not through a transcriptional mechanism. Instead, the Drosophila transcript also has a 5' UTR with uORFs that trans- lationally activates this gene upon elF2alpha phosphorylation (Fig. 2A). Once synthesized, PPP1R15 opposes PERK's effect on elF2alpha (49).

Independent studies have reported the presence of ATF4-like 5' UTRs in a number of other transcripts. These include ATF5 and CHOP (C/EBP homologous protein), transcription factors that contribute to the UPR (50), as well as a kinase of unclear function $(51,52)$. As there have not been any systematic efforts to identify such transcripts in Drosophila, it is likely that additional transcripts specifically translated during UPR remain at large.

\section{ROLE OF THE PERK/ATF4 PATHWAY IN Drosophila DEVELOPMENT}

A hypomorphic allele of ATF4, cryptocephal', was first described in the 1940s as a mutant with heads that fail to emerge from the thorax during the pupal stage of development (53). Null alleles show defects in larval molting and pupariation (46). Consistent with this idea, Drosophila ATF4 transcriptionally induces Ecdysis triggering hormone $(\mathrm{ETH})$ in the endocrine cells, which in turn promote molting. Intriguingly, a recent study reported that ATF4 works as a coactivator of the Ecdysone receptor, which controls numerous target genes involved in metamorphosis (54). As ATF4 protein cannot be synthesized without stress-induced elF2alpha phosphorylation, these observations provide a tantalizing link between metamorphosis and ER stress, which remains to be further characterized.

Consistent with the developmentally essential role of ATF4, we recently reported that the PERK allele e01744, with a Piggybac element inserted in the first intron, causes developmental lethality. Moreover, PERK is active in healthy adult intestinal stem cells, and without PERK, intestinal stem cell proliferation is reduced. Intriguingly, PERK activity in the stem cells has negative consequences on the intestinal epithelium, and knock down of PERK in that tissue prolongs lifespan of Drosophila (37). These observations show that the PERK/ATF4 pathway is also active in healthy tissues. While it plays positive roles in certain tissues as judged by their developmental requirement, the pathway can have negative effects in others.

In mammals, there are a few additional layers of complexity to this pathway. One is the fact that there are four elF2alpha kinases, each mediating distinct stress response. Two of those, PERK and GCN2, are conserved in Drosophila (49) (Fig. 2A). GCN2 is specifically activated by amino acid deprivation, and consistent with this, ATF4's transcriptional targets include amino acid transporters and other metabolic genes (55-57). Adding to the complexity are non-canonical downstream effectors of PERK. Specifically, studies have reported the transcription factors Nrf2 and NF-kappaB to lie downstream of PERK (58-60). Whether these non-canonical axes of PERK signaling also exist in Drosophila, and whether they play physiologically significant roles in vivo remains to be determined. 


\section{THE ATF6 PATHWAY OF THE UPR}

In vertebrates, ATF6 also plays an important role in the UPR. This protein has a DNA binding domain in addition to a transmembrane domain that tethers the protein at the ER membrane under unstressed conditions. Upon stress, ATF6 is released from the ER and traffics to the Golgi, where it is cleaved by membrane associated proteases to release the cytoplasmic portion $(61,62)$. Such conditions allow ATF6 to translocate to the nucleus, where it induces many ER quality control genes including XBP1 and ER chaperones $(63,64)$. There are two ATF6 genes in vertebrates, referred to as ATF6alpha and beta. Single knockout mice are viable, but double knockouts are embryonic lethal, indicative of redundancy of function in the two genes (64).

The Drosophila genome encodes a single ATF6 homolog, with conservation not only within the DNA binding domain, but also in the transmembrane and luminal domains. A Piggybac insertion line within the coding sequence of ATF6, PBac LL0743, is available from public stock centers. This insertion line is viable and fertile as homozygotes (unpublished data), indicating that Drosophila atf6 does not play a developmentally essential role as in mammals. Whether this gene is required for a proper ER stress response in adult tissues remain to be validated.

\section{THE UPR IN Drosophila DISEASE MODELS}

The role of IRE1 and XBP1 in vivo has been studied most extensively in the context of mutations that impair the folding property of cells in the ER. A well-characterized example is the Drosophila ninaE mutant alleles that cause age-related photoreceptor degeneration $(65,66)$. Drosophila ninaE encodes a light detecting protein, Rhodopsin-1, and certain missense mutant alleles are similar in nature with the human rhodopsin alleles that underlie Autosomal Dominant Retinitis Pigmentosa (ADRP) (67). Although the human alleles have been speculated to impair the encoded protein's folding property, the link between the UPR and retinal degeneration by rhodopsin mutants was first established through the study of the Drosophila nina $E^{\mathrm{C} 69 D}$ mutant. Specifically, the XBP1-GFP reporter was used to show that the IRE1/XBP1 pathway is activated in these mutant photoreceptors, and that the loss of XBP1 accelerated the course of degeneration in this disease model (24). Conversely, enhancing the degradation of misfolded Rhodopsin-1, by overexpressing the HRD1 ubiquitin ligase, suppressed the course of retinal degeneration in Drosophila (68). In human ADRP, P23H substitution is the most common mutation, and the equivalent mutant $\mathrm{P} 37 \mathrm{H}$ was generated in the fly to establish similar UPR activation (69).

It appears that Drosophila Rhodopsin-1 is particularly sensitive to the protein-folding environment in the ER. Drosophila genome encodes two Calnexins. Mutation in one of those, Calnexin 99A, gives rise to viable adults with significantly re- duced Rhodopsin-1 levels in their photoreceptors (70). A number of other genetic conditions impair proper rhodopsin folding in the ER. Most recently, it has been found that a complex of proteins with previously unknown function, termed the ER membrane protein complex (EMC), are required for multipass transmembrane domain protein folding. In photoreceptors, loss of EMC subunits resulted in Rhodopsin-1 misfolding (71). Although excessive ER stress is a cause of cellular dysfunction and cell death, it has been reported that there are milder conditions of rhodopsin misfolding can protect cells from other kinds of stress. Such effect was observed while examining p53-induced photoreceptor cell death. The authors found that a mutation in ninaA, a gene that is normally required for proper Rhodopsin-1 folding, protects against p53-induced cell death (72). The degree of rhodopsin misfolding is probably mild enough to avoid photoreceptor degeneration under these conditions, while activating UPR's ability to attenuate translation and enhance anti-oxidant response to enhance general stress resistance of cells.

UPR is associated with a number of other neurodegenerative diseases. Research in Drosophila established a link between IRE1/XBP1 and a VapB mutation that underlies amyotrophic lateral sclerosis. VapB is a transmembrane protein with an immunoglobulin fold domain, MSB. Drosophila encodes a homologous gene, VAP, whose loss results in the disruption of neuromuscular junctions (73). Interestingly, a point mutation in the human gene, P56S, underlies the dominant effects in the motor neuron degeneration of amyotrophic lateral sclerosis (74), but the underlying reason for pathogenesis had remained unclear, before Drosophila was employed as a model system. Studies have determined that the P56S equivalent mutation in Drosophila, P58S, forms aggregates in the ER and activates the IRE1/XBP1 pathway of the UPR $(75,76)$. Defects in the ER quality control in this model are partly attributed to a failure of mutant VapB to bind and retain Oxysterol binding protein in the ER (76). In addition, this mutant allele shows a non-autonomous effect, as wild type VAP has an MSB domain that is cleaved off for secretion to bind to Ephrin reeceptors in neighboring cells, which does not occur in the mutants (75).

Another example of disease associated with UPR is Hereditary spastic paraplegias. These are neurological disorders that show progressive stiffness and spasticity in the lower limbs, due to damages or dysfunction of nerve fibers. A mutation in a reticulon family protein, RTN2, underlies an autosomal dominant form of this disease. Drosophila encodes a single reticulon, Rtn1. A recent study has found that the protein product is enriched in axons and is essential for proper organization of smooth ER in the distal parts of axons. Rtn1 mutants activate the UPR, as evidenced by XBP1-GFP expression (77).

Mutations in the cytoplasmic aminoacyl-tRNA synthetases are associated with Charcot-Marie-Tooth diseases, a common form of neurological disorder. A recent study has examined the physiological defects associated with phenylalanyl-tRNA synthetase mutations that underlie this disease, and found that, 
among others, the mutant protein expression triggers the activation of XBP1-GFP, providing a link between CharcotMarie-Tooth disease and UPR (78). Very recently, a genetic screen for genes involved in axonal regeneration after injury identified regulators of XBP1 mRNA splicing. The study found that loss of XBP1 reduces axonal regeneration, whereas conditions that enhance XBP1 splicing stimulates it (79).

In a number of genetic conditions, it has been shown that hyper-stimulation of the IRE1/XBP1 pathway helps to suppress phenotypes. An interesting examples have been reported in a Drosophila model for Alzheimer's disease, in which the amyloid-beta 1-42 peptide or mutant tau were overexpressed in the developing eye $(80,81)$. Such conditions not only activated the XBP1-GFP reporter, but overexpressing the spliced isoform of XBP1 suppressed the phenotype caused by amyloid-beta expression (81). Spliced XBP1 overexpression can also suppress a distinct phenotype associated with the disruption of the ER-mitochondria interface, caused by the knock down of mitofusin (82).

Drosophila has been actively employed to express human gene alleles that underlie diseases. Among those associated that activate the UPR include mutations in Pro-insulin that underlie diabetes (83), alpha-one antitrypsin mutations that underlie lung emphesyma $(68,84)$, the GBA gene that underlies Gaucher disease (85).

\section{OTHER UPR PATHWAYS}

Most studies in the field focus on the three UPR branches, mediated by IRE1, PERK and ATF6, respectively. These pathways are fully activated by ER stress within hours, and quickly become inactivated through feedback mechanisms. Although a number of studies have implicated their role in degenerative diseases, it is difficult to imagine how these pathways can be responsible for age-related degenerative diseases that manifest only after decades of chronic ER stress. Studies from Drosophila point to the role of distinct pathways that contribute to ER-stress inducible cell death, possibly involving $\mathrm{Ca}^{2+}$. Notably, the ER is a major storage site for $\mathrm{Ca}^{2+}$, and excessive leakage into the cytoplasm can trigger cell death.

Interestingly, an RNAi screen for genes that are required for mutant Rhodopsin-1 induced cell death identified stress-activated kinases, CDK5 and MEKK1, which had not been previously implicated in the canonical UPR pathways (47). CDK5 is a kinase that can be activated by ROS or excessive $\mathrm{Ca}^{2+}$ in the cytoplasm, and implicated in other forms of neuronal cell death (86). Interestingly, excessive $\mathrm{Ca}^{2+}$ release into the cytoplasm also occurs in a Drosophila model for Alzheimer's disease, where amyloid beta peptide overexpression imposes stress in the ER. The amyloid beta overexpression phenotype is suppressed in the mutant background of Ryanodine Receptor, whose normal role is to release $\mathrm{Ca}^{2+}$ from the ER to the cytoplasm (81). Based on this, one can put together a working hypothesis that chronic stress in the ER causes $\mathrm{Ca}^{2+}$ to leak out into the cytoplasm, and initiate a distinct signaling pathway mediated by CDK5 and MEKK1. This particular signaling pathway may better explain gene expression changes that occur after decades of chronic ER stress, as breakdown of ER $\mathrm{Ca}^{2+}$ homeostasis may occur possibly years after chronic exposure to ER stress, whereas PERK, IRE1 and ATF6 mediate acute UPR responses within hours.

\section{CONCLUSION}

UPR research in Drosophila has accelerated in recent years, in part due to the availability of new genetic tools. This model organism nicely complements the existing approaches, particularly in the investigation of the normal physiological role of the UPR, and also regarding specific disease mechanisms. Regarding the molecular mechanism of UPR, much progress has been made in the IRE1/XBP1 branch of signaling. Our understanding of the PERK/ATF4 pathway in Drosophila has been gradually improving, but research on the other branches still lag behind. Unbiased genetic approaches in Drosophila may help elucidate other branches of the UPR that may remain at large.

\section{ACKNOWLEDGEMENTS}

I thank Brian Brown for comments on the manuscript. This work was supported by the NIH grant R01 EY020866, and the March of Dimes grant FY13-204.

\section{REFERENCES}

1. Ghaemmaghami S, Huh WK, Bower K et al (2003) Global analysis of protein expression in yeast. Nature 425, 737-741

2. Kanapin A, Batalov S, Davis MJ et al (2003) Mouse proteome analysis. Genome Res 13, 1335-1344

3. Braakman I, Hebert DN (2013) Protein folding in the endoplasmic reticulum. Cold Spring Harb Perspect Biol 5, a013201

4. Marciniak SJ, and Ron D (2006) Endoplasmic reticulum stress signaling in disease. Physiol Rev 86, 1133-1149

5. Hetz C, Mollereau B (2014) Disturbance of endoplasmic reticulum proteostasis in neurodegenerative diseases. Nat Rev Neurosci 15, 233-249

6. Kozutsumi Y, Segal M, Normington K, Gething MJ, Sambrook J (1988) The presence of malfolded proteins in the endoplasmic reticulum signals the induction of glucose-regulated proteins. Nature 332, 462-464

7. Walter P, Ron D (2011) The unfolded protein response: from stress pathway to hemeostatic regulation. Science $334,1081-1086$

8. Lytton J, Westlin M, Hanley MR (1991) Thapsigargin inhibits the sarcoplasmic or endoplasmic reticulum Ca-ATPase family of calcium pumps. J Biol Chem 266, 17067-17071

9. Trombetta ES, Helenius A (1998) Lectins as chaperones in 
glycoprotein folding. Curr Opin Struct Biol 8, 587-592

10. Bulleid NJ, van Lith M (2014) Redox regulation in the endoplasmic reticulum. Biochem Soc Trans 42, 905-908

11. Chow CY, Wolfner MF, Clark AG (2013) Using natural variation in Drosophila to discover previously unknown endoplasmic reticulum stress genes. Proc Natl Acad Sci U S A 110, 9013-9018

12. Chapman R, Sidrauski C, Walter P (1998) Intracellular signaling from the endoplasmic reticulum to the nucleus. Annu Rev Cell Dev Biol 14, 459-485

13. Cox JS, Shamu CE, and Walter P (1993) Transcriptional induction of genes encoding endoplasmic reticulum resident proteins requires a transmembrane protein kinase. Cell 73, 1197-1206

14. Korennykh AV, Egea PF, Korostelev AA et al (2009) The unfolded protein response signals through higher-order assembly of Ire1. Nature 457, 687-693

15. Lee KP, Dey M, Neculai D, Cao C, Dever TE, Sicheri F (2008) Structure of the dual enzyme ire1 reveals the basis for catalysis and regulation in nonconventional RNA splicing. Cell 132, 89-100

16. Sidrauski C, Cox JS, Walter P (1996) tRNA ligase is required for regulated mRNA splicing in the unfolded protein response. Cell 87, 405-413

17. Travers KJ, Patil CK, Wodicka L, Lockhart DJ, Weissman JS, Walter P (2000) Functional and genomic analyses reveal an essential coordination between the unfolded protein response and ER-associated degradation. Cell 101, 249-258

18. Wang $X Z$, Harding HP, Zhang $\mathrm{Y}$, Jolicoeur EM, Kuroda $M$, and Ron D (1998) Cloning of mammalian Ire1 reveals diversity in the ER stress responses. EMBO J 17, 5708-5717

19. Tirasophon W, Welihinda AA, and Kaufman RJ (1998) A stress response pathway from the endoplasmic reticulum to the nucleus requires a novel bifunctional protein kinase/endoribonuclease (Ire1p) in mammalian cells. Genes Dev 12, 1812-1824

20. Lu Y, Liang FX, Wang X (2014) A synthetic biology approach identifies the mammalian UPR RNA ligase RtcB. Mol Cell 55, 758-770

21. Jurkin J, Henkel T, Nielsen AF et al (2014) The mammalian tRNA ligase complex mediates splicing of XBP1 mRNA and controls antibody secretion in plasma cells. EMBO J 33, 2922-2936

22. Kosmaczewski SG, Edwards TJ, Han SM et al (2014) The RtcB RNA ligase is an essential component of the metazoan unfolded protein response. EMBO Rep 15, 1278-1285

23. Hollien J, and Weissman JS (2006) Decay of endoplasmic reticulum-localized mRNAs during the unfolded protein response. Science 313, 104-107

24. Ryoo HD, Domingos PM, Kang MJ, and Steller H (2007) Unfolded protein response in a Drosophila model for retinal degeneration. EMBO J 26, 242-252

25. Lisbona F, Rojas-Rivera D, Thielen P et al (2009) BAX inhibitor-1 is a negative regulator of the ER stress sensor IRE1alpha. Mol Cell 33, 679-691

26. Niwa M, Patil CK, DeRisi J, and Walter P (2005) Genome-scale approaches for discovering novel nonconventional splicing substrates of the Ire1 nuclease.
Genome Biol 6, R3

27. Gaddam D, Stevens N, Hollien J (2013) Comparison of mRNA localization and regulation during endoplasmic reticulum stress in Drosophila cells. Mol Biol Cell 24, 14-20

28. Coelho DS, Cairrão F, Zeng X et al (2013) Xbp1-independent Ire1 signaling is required for photoreceptor differentiation and rhabdomere morphogenesis in Drosophila. Cell Rep 14, 791-801

29. Han D, Lerner AG, Vande Walle L et al (2009) IIRE1alpha kinase activation modes control alternate endoribonuclease outputs to determine divergent cell fates. Cell 138, 562-572

30. Hollien J, Lin JH, Li H, Stevens N, Walter P, Weissman JS (2009) Regulated Ire1-dependent decay of messenger RNAs in mammalian cells. J Cell Biol 186, 323-331

31. Kimmig P, Diaz M, Zheng J et al (2012) The unfolded protein response in fission yeast modulates stability of select mRNAs to maintain protein homeostasis. Elife 1, e00048

32. Ryoo HD, Li J, Kang MJ (2013) Drosophila XBP1 expression reporter marks cells under endoplasmic reticulum stress and with high protein secretory load. PLoS One 8, e75774

33. Arsham AM, Neufeld TP (2009) A genetic screen in Drosophila reveals novel cytoprotective functions of the autophagy-lysosome pathway. PLoS One 4, e6068

34. Sone M, Zeng X, Larese J, Ryoo HD (2013) A modified UPR stress sensing system reveals a novel tissue distribution of IRE1/XBP1 activity during normal Drosophila development. Cell Stress Chaperones 18, 307-319

35. Wang L, Zeng X, Ryoo HD, Jasper H (2014) Integration of UPRER and oxidative stress signaling in the control of intestinal stem cell proliferation. PLoS Genet 10, e1004568

36. Chow CY, Avila FW, Clark AG, Wolfner MF (2015) Induction of excessive endoplasmic reticulum stress in the Drosophila male accessory gland results in infertility. PLoS One 10, e0119386

37. Wang L, Ryoo HD, Qi Y, Jasper H (2015) PERK limits Drosophila lifespan by promoting intestinal stem cell proliferation in response to systemic and local ER stress. PLoS Genet 11, e1005220

38. Tien AC, Rajan A, Schulze KL et al (2008) Ero1L, a thiol oxidase, is required for Notch signaling through cysteine bridge formation of the Lin12-Notch repeats in Drosophila melanogaster. J Cell Biol 182, 1113-1125

39. Zito E, Chin KT, Blais J, Harding HP, Ron D (2010) ERO1-beta, a pancreas-specific disulfide oxidase, promotes insulin biogenesis and glucose homeostasis. J Cell Biol 188, 821-832

40. Groth C, Sasamura T, Khanna MR, Whitley M, Fortini ME (2013) Protein trafficking abnormalities in Drosophila tissues with impaired activity of the ZIP7 zinc transporter Catsup. Development 140, 3018-3027

41. Acar M, Jafar-Nejad H, Takeuchi $\mathrm{H}$ et al (2008) Rumi is a CAP10 domain glycosyltransferase that modifies Notch and is required for Notch signaling. Cell 132, 247-258

42. Yamakawa T, Yamada K, Sasamura T et al (2012) Deficient Notch signaling associated with neurogenic pecanex is compensated for by the unfolded protein response in Drosophila. Development 139, 558-567

43. Harding HP, Zhang Y, Ron D (1999) Protein translation 
and folding are coupled by an endoplasmic-reticulum-resident kinase. Nature 397, 271-274

44. Malzer E, Daly ML, Moloney A et al (2010) Impaired tissue growth is mediated by checkpoint kinase 1 (CHK1) in the integrated stress response. J Cell Sci 123, 2892-2900

45. Dever TE, Feng L, Wek RC, Cigan AM, Donahue TF, Hinnebusch AG (1992) Phosphorylation of initiation factor 2 alpha by protein kinase GCN2 mediates gene-specific translational control of GCN4 in yeast. Cell 68, 585-596

46. Hewes RS, Schaefer AM, Taghert PH (2000) The cryptocephal gene (ATF4) encodes multiple basic-leucine zipper proteins controlling molting and metamorphosis in Drosophila. Genetics 155, 1711-1723

47. Kang MJ, Chung J, Ryoo HD (2012) CDK5 and MEKK1 mediate pro-apoptotic signalling following endoplasmic reticulum stress in an autosomal dominant retinitis pigmentosa model. Nat Cell Biol 14, 409-415

48. Kang K, Ryoo HD, Park JE, Yoon JH, Kang MJ (2015) A Drosophila reporter for the translational activation of ATF4 marks stressed cells during development. PLoS One 10, e0126795

49. Malzer E, Szajewska-Skuta M, Dalton LE et al (2013) Coordinate regulation of elF2alpha phosphorylation by PPP1R15 and GCN2 is required during Drosophila development. J Cell Sci 126, 1406-1415

50. Palam LR, Baird TD, Wek RC (2011) Phosphorylation of elF2 facilitates ribosomal bypass of an inhibitory upstream ORF to enhance CHOP translation. J Biol Chem 286, 10939-10949

51. Watatani Y, Ichikawa K, Nakanishi N et al (2008) Stress-induced translation of ATF5 mRNA is regulated by the 5' untranslated region. J Biol Chem 283, 2543-2553

52. Baird TD, Palam LR, Fusakio ME et al (2014) Selective mRNA translation during elF2 phosphorylation induces expression of IBTKalpha. Mol Biol Cell 25, 1686-1697

53. Hadorn E, Gloor H (1943) Cryptocephal, ein spat wirkender Leftalfaktor bei Drosophila melanogaster. Rev Suisse Zool 50, 256-261

54. Gaithier SA, VanHaaften E, Cherbas L, Cherbas P, Hewes RS (2012) Cryptocephal, the Drosophila melanogaster ATF4, is a specific coactivator for ecdysone receptor isoform B2. PLoS Genet 8, e1002883

55. Harding HP, Zhang Y, Zeng $\mathrm{H}$ et al (2003) An integrated stress response regulates amino acid metabolism and resistance to oxidative stress. Mol Cell 11, 619-633

56. Han J, Back SH, Hur J et al (2013) ER-stress-induced transcriptional regulation increases protein synthesis leading to cell death. Nat Cell Biol 15, 481-490

57. Lee JE, Oney M, Frizzell K, Phadnis N, Hollien J (2015) Drosophila melanogaster Activating Transcription Factor 4 regulates glycolysis during endoplasmic reticulum stress. G3 (Bethesda) 5, 667-675

58. Cullinan SB, Zhang D, Hannink M, Arvisais E, Kaufman RJ, Diehl JA (2003) Nrf2 is a direct PERK substrate and effector of PERK-dependent cell survival. Mol Cell Biol 23, 7198-7209

59. Jiang HY, Wek SA, McGrath BC et al (2003) Phosphorylation of the alpha subunit of eukaryotic initiation factor 2 is required for activation of NF-kappaB in response to diverse cellular stresses. Mol Cell Biol 23, 5651-5663

60. Deng J, Lu PD, Zhang Y et al (2004) Translational repression mediates activation of nuclear factor kappa B by phosphorylated translational initiation factor 2. Mol Cell Biol 24, 10161-10168

61. Ye J, Rawson RB, Komuro R et al (2000) ER stress induces cleavage of membrane-bound ATF6 by the same proteases that process SREBPs. Mol Cell 6, 1355-1364

62. Shen J, Chen X, Hendershot L, and Prywes R (2002) ER stress regulation of ATF6 localization by dissociation of BiP/GRP78 binding and unmasking of Golgi localization signals. Dev Cell 3, 99-111

63. Yoshida H, Okada T, Haze K et al (2001) Endoplasmic reticulum stress-induced formation of transcription factor complex ERSF including NF-Y (CBF) and activating transcription factors 6alpha and 6beta that activates the mammalian unfolded protein response. Mol Cell Biol 21, 1239-1248

64. Yamamoto K, Sato T, Matsui T et al (2007) Transcriptional induction of mammalian ER quality control proteins is mediated by single, or combined action of ATF6alpha and xbp1. Dev Cell 13, 365-376

65. Kumar JP, Ready DF (1995) Rhodopsin plays an essential structural role in Drosophila photoreceptor development. Development 121, 4359-4370

66. Colley NJ, Cassill JA, Baker EK, Zuker CS (1995) Defective intracellular transport is the molecular basis of rhodopsin-dependent dominant retinal degeneration. Proc Natl Acad Sci U S A 92, 3070-3074

67. Sung CH, Davenport CM, Hennessey JC et al (1991) Rhdopsin mutations in autosomal dominant retinitis pigmentosa. Proc Natl Acad Sci U S A 88, 6481-6485

68. Kang MJ, Ryoo HD (2009) Suppression of retinal degeneration in Drosophila by stimulation of ER-Associated Degradation. Proc Natl Acad Sci U S A 106, 17043-17048

69. Griciuc A, Aron L, Roux MJ, Klein R, Giangrande A, Ueffing M (2010) Inactivation of VCP/ter94 suppresses retinal pathology caused by misfolded rhodopsin in Drosophila. PLoS Genet 6, e1001075

70. Rosenbaum EE, Hardie RC, Colley NJ (2006) Calnexin is essential for Rhodopsin maturation, $\mathrm{Ca} 2+$ regulation, and photoreceptor cell survival. Neuron 49, 229-241

71. Satoh T, Ohba A, Liu Z, Inagaki T, Satoh AK (2015) $\mathrm{dPob} / \mathrm{EMC}$ is essential for biosynthesis of rhodopsin and other multi-pass membrane proteins in Drosophila photoreceptors. Elife 4, e06306

72. Mendes CS, Levet C, Chatelain G et al (2009) ER stress protects from retinal degeneration. EMBO J 28, 1296-1307

73. Pannetta G, Hiesinger PR, Fabian-Fine R, Meinertzhagen IA, Bellen HJ (2002) Drosophila VAP-33A directs bouton formation at neuromuscular junctions in a dosage-dependent manner. Neuron 35, 291-306

74. Nishimura AL, Mitne-Neto M, Silva HC et al (2004) A mutation in the vesicle-trafficking protein VAPB causes late-onset spinal muscular atrophy and amyotrophic lateral sclerosis. Am J Hum Genet 75, 822-831

75. Tsuda H, Han SM, Yang Y et al (2008) The amyotriphic lateral sclerosis 8 protein VAPB is cleaved, secreted, and 
acts as a ligand for Eph receptors. Cell 133, 963-977

76. Moustaqim-Barrette A, Lin YQ, Pradhan S, Neely GG, Bellen HJ, Tsuda H (2014) The amyotrophic lateral sclerosis 8 protein, VAP, is required for ER protein quality control. Hum Mol Genet 23, 1975-1989

77. O'Sullivan NC, Jahn TR, Reid E, O'Kane CJ (2012) Reticulon-like-1, the Drosophila orthologue of the hereditary spastic paraplegia gene reticulon 2, is required for organization of endoplasmic reticulum and of distal motor axons. Hum Mol Genet 21, 3356-3365

78. Lu J, Bergert M, Walther A, Suter B (2014) Double-sieving-defective aminoacyl-tRNA synthetase causes protein mistranslation and affects cellular physiology and development. Nat Commun 5, 5650

79. Song Y, Stretavan D, Salegio EA et al (2015) Regulation of axon regeneration by the RNA repair and splicing pathway. Nat Neurosci 18, 817-825

80. Loewen CA, Feany MB (2010) The unfolded protein response protects from tau neurotoxicity in vivo. PLoS One 5, e13084

81. Casas-Tinto S, Zhang Y, Sanchez-Garcia J, Gomez-
Velazquez $M$, Rincon-Limas DE, Fernandez-Funez $P$ (2011) The ER stress factor XBP1s prevents amyloid-beta neurotoxicity. Hum Mol Genet 20, 2144-2160

82. Debattisti V, Pendin D, Ziviani E, Daga A, Scorrano L (2014) Reduction of endoplasmic reticulum stress attenuates the defects caused by Drosophila mitofusin depletion. J Cell Biol 204, 303-312

83. Park SY, Ludwig MZ, Tamarina NA et al (2014) Genetic complexity in a Drosophila model of diabetes-associated misfolded human proinsulin. Genetics 196, 539-555

84. Jang BY, Ryoo HD, Son J et al (2015) Role of Drosophila EDEMs in the degradation of the alpha-1-antitrypsin Z variant. Int J Mol Med 35, 870-876

85. Suzuki T, Shimoda M, Ito K et al (2013) Expression of human gaucher disease gene GBA generates neurodevelopmental defects and ER stress in Drosophila eye. PLoS One 8, e69147

86. Qu D, Rashidian J, Mount MP et al (2007) Role of Cdk5-mediated phosphorylation of Prx2 in MPTP toxicity and Parkinson's disease. Neuron 55, 37-52 